\section{Chaperoning extended life}

The capacity to moderate internal and external stress is arguably the central function regulating senescence in whole-animal ageing $^{1-3}$. During ageing, molecular chaperones such as heat-shock proteins are thought to combat stress-related senescent dysfunction $^{4,5}$. In transgenic Drosophila melanogaster, with varying copy numbers of the gene $h s p 70$ encoding heat-shock protein hsp70, we found that heat-induced expression of hsp70 increased lifespan at normal temperatures. Only a brief, low level of expression was required to obtain a longterm improvement in survival.

Thermally conditioned D. melanogaster and Caenorhabditis elegans exhibit greater longevity when mildly heated adults are returned to normal temperatures ${ }^{6,7}$. In $D$. melanogaster, such extended longevity is caused by an immediate increase in agespecific survival that persists for several weeks ${ }^{6}$. This change in survival is not easily explained by the removal of mortality costs of reproduction because these levels of thermal conditioning do not reduce egg laying. Rather, improved longevity may represent the modulation of age-related stress by the induced expression of heatshock proteins.

We used transgenic D. melanogaster strains (from S. Lindquist), which varied in the number of copies of the inducible $h s p 70$ gene $^{8,9}$, to determine the effect of hsp70 protein on survival during ageing at normal temperatures. These allelic strains control

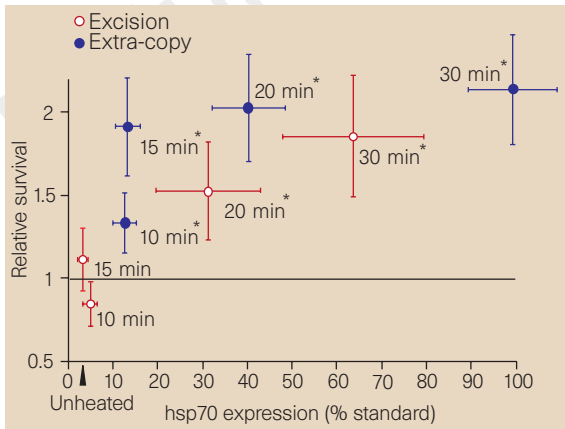

Figure 1 Effect of hsp70 expression on relative survival in 'extra-copy' and 'excision' strains of Drosophila. Within each strain, relative survival is calculated over the 14 days after heat shock compared with a control cohort of unheated flies (see ref. 6). Life tables were constructed from cohorts of about 1,200 flies per treatment. Heat treatments varied from 10 to $30 \mathrm{~min}$, and durations that produced significant increases in survival are marked with asterisks. Levels of hsp70 protein were measured by enzyme-linked immunosorbent assay (ELISA) ${ }^{9}$. Arrow shows the background hsp70 level in unheated flies. Standard errors are shown for estimates of both variables. for the effects of insertional mutagenesis. The 'excision' strain carried the wild-type complement of constitutive $h s p 70$ and heatinducible $h s p 70$ genes. The 'extra-copy' strain added to the second chromosome a total of 12 additional copies of heatinducible $h s p 70$. The excision strain carried only a remnant P-element construct at the same integration site.

Four days after emergence of adult males, we heat-treated each strain with a brief, non-lethal $36{ }^{\circ} \mathrm{C}$ pulse of varying duration, and measured the subsequent expression of induced hsp70 protein, agespecific survival and remaining life expectancy at $24^{\circ} \mathrm{C}$. In independent trials we replicated these results, characterized the time-course of hsp70 protein induction, and observed the effects of longer heat treatments.

The presence of hsp70 protein increased subsequent survival at normal temperatures when expression exceeded $10-12 \%$ of standard (Fig. 1). The improvement in survival reached a plateau with higher levels of expressed protein. 'Extra-copy' but not 'excision' flies heated for 10 or $15 \mathrm{~min}$ expressed hsp70 and had improved survival over the two-week period after heat shock. Under these conditions, life expectancy increased by as much as $7.9 \%$ in 'extracopy' flies, which is a substantial rise given the prevailing low mortality rates of young adults. Short heat treatments failed to induce hsp70 or to improve survival in the excision flies. These observations show that hsp70 moderates survival during subsequent ageing.

Heat treatments of 20 and $30 \mathrm{~min}$ induced hsp70 to levels greater than $30 \%$ of standard in both strains and improved survival by similar amounts in each. Because the relationship between improved survival and hsp70 is similar in 'extra-copy' and 'excision' strains, we infer that the strains differ solely in their ability to express hsp70 protein for a given heat dose. This functional similarity also suggests that $h s p 70$ copy number, and not merely differences in insert size, causes the improved survival after heat shock.

The whole-fly titre of induced hsp70 protein is transient, but its effect on agespecific survival is persistent. A transient expression of a molecular chaperone may increase age-specific survival through its ability to renature, assemble and disassemble many non-heat-shock proteins ${ }^{10}$, and to interact with other stress-response mechanisms such as superoxide dismutase ${ }^{5}$. It is also possible that hsp70 persists at high levels in some critical and specific tissue over the period of increased survival. Although hsp70 and heat-shock proteins in general are tightly regulated ${ }^{10}$, transient but effective levels of hsp70 could be present when stress is routinely encountered, and hsp70 may repair and restore higher order cell functions which themselves would otherwise accelerate senescence.

Marc Tatar*, Aziz A. Khazaeli

James W. Curtsinger

Department of Ecology, Evolution and Behavior,

University of Minnesota, St Paul,

Minnesota 55108, USA

e-mail: Marc_Tatar@Brown.edu

${ }^{*}$ Present address: Department of Ecology and Evolutionary

Biology, Box G-W, Brown University, Providence, Rhode Island 02912, USA.

1. Jazwinski, S. M. Science 273, 54-59 (1996).

2. Lithgow, G. J. BioEssays 18, 809-815 (1996).

3. Murakami, S. \& Johnson, T. E. Genetics 143, 1207-1218 (1996).

4. Heydari, A. R., Takahashi, T., Gutsmann, A., You, S. \& Richardson, A. Experientia 50, 1092-1098 (1994).

5. Wheeler, J. C., Bieschke, E. T. \& Tower, J. Proc. Natl Acad. Sci. USA 92, 10408-10412 (1995).

6. Khazaeli, A. A., Tatar, M., Pletcher, S. D. \& Curtsinger, J. W. J. Gerontol. A 52, B48-B52 (1997).

7. Lithgow, G. J., White, T. M., Melov, S. \& Johnson, T. E. Proc. Natl Acad. Sci. USA 92, 7540-7544 (1995).

8. Welte, M. A., Tetrault, J. M., Dellavalle, R. P. \& Lindquist, S. L. Curr. Biol. 13, 842-853 (1993).

9. Feder, M. E., Cartano, N. V., Milos, L., Krebs, R. A. \& Lindquist, S. L. J. Exp. Biol. 119, 1837-1844 (1997).

10. Parsell, D. A. \& Lindquist, S. Annu. Rev. Genet. 27, 437-496 (1993).

\section{Probabilities of}

\section{conspecificity}

G. Suwa et al. ${ }^{1}$ and E. Delson's News and Views ${ }^{2}$ address one of the most contentious issues in palaeoanthropology, that of the boundaries (if any) between hominid species, in the context of an exciting new fossil from Konso in Ethiopia. Suwa et al. attribute this specimen to Australopithecus boisei, but note that it has some similarities to the South African robust australopithecines first described by Robert Broom more than 50 years ago.

As the Nature authors say, there is certainly a need to be careful about 'pigeonholing' particular fossils into a particular species. One way of addressing this issue is to try to assess probabilities of conspecificity when pairs of specimens are compared. By using a simple morphometric technique based on comparison of anatomical measurements of conspecific pairs of extant taxa (1,260 specimens, representing 70 vertebrate and invertebrate species), we have found a lognormal distribution of the standard error of the $m$-coefficient associated with equations of the general form $y=m x+c$ derived from linear regression analysis $^{3}$. The method has been used in comparisons between the type specimens of two 'robust' australopithecines, OH 5 ( $A$. boisei from Olduvai Gorge in Tanzania, described by P. V. Tobias), and TM 1517 (A. robustus, from Kromdraai in South Africa), suggesting a high probability of conspecificity. The $\log$ standard error value of -1.31 determined by comparing cranial 
dimensions measurable from both specimens is within two standard deviations of the mean value of -1.78 obtained from conspecific comparisons of large samples of extant vertebrate and invertebrate taxa ${ }^{3}$.

Although this result suggests that the type specimens of $A$. robustus and $A$. boisei have a high probability of conspecificity, this does not mean that all specimens attributed to A. boisei should necessarily be referred to as $A$. robustus. As a South African in the new South Africa, I believe we should move away from rigid application of the binomial system of classification in palaeontological contexts.

\section{J. F. Thackeray}

Transvaal Museum, PO Box 413,

Pretoria 0001, South Africa

1. Suwa, G. et al. Nature 389, 489-492 (1997).

2. Delson, E. Nature 389, 445-446 (1997).

3. Thackeray, J. F. et al. S. Afr. J. Sci. 93, 195-196 (1997).

\section{Xenoturbella's}

\section{molluscan relatives...}

Despite detailed morphological studies ${ }^{1-4}$, the phylogenetic relationships of Xenoturbella bocki Westblad 1949 have remained unclear. The marine, worm-like X. bocki was first described as an acoel flatworm ${ }^{5}$. Later it was proposed to be a deuterostome $^{1}$, and most recently as the sister taxon of the Bilateria ${ }^{6}$. Here we present DNA sequence data that place $X$. bocki within the protostome clade Eutrochozoa.

We used standard DNA extraction, polymerase chain reaction (PCR) and sequencing techniques to sequence 1,759 nucleotides of the small-subunit ribosomal RNA gene (18S rRNA) and 708 base pairs of the protein-coding mitochondrial cytochrome $c$ oxidase subunit I gene (COI) from five specimens of $X$. bocki collected on the west coast of Sweden. We sequenced the corresponding COI fragment from the flatworm Graffilla buccinicola for comparison. We used these and sequences obtained from GenBank to construct two matrices for cladistic analysis.

The 18S rRNA matrix (778 informative characters) comprised 47 sequences from 18 phyla relevant to the position of $X$. bocki of which 35 sequences were previously aligned according to secondary structure ${ }^{7}$. The remaining sequences were added using ClustalW $^{8}$. There was less COI data - the COI matrix (370 informative characters) comprised 22 taxa from 8 phyla.

We used parsimony jack-knifing (resampling with deletion of a fraction of the characters) to generate phylogenetic hypotheses and to evaluate their support (using prerelease 'Xac' software, J. S. Farris) ${ }^{9}$. To eliminate homoplasious nucleotide positions in

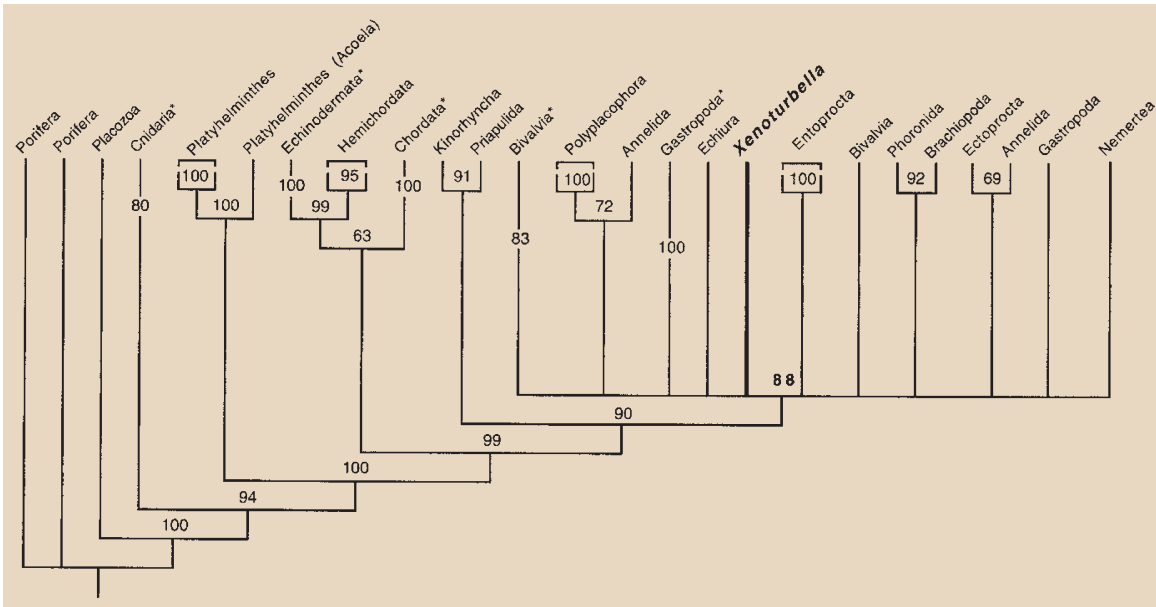

Figure 1 Consensus tree showing groups present in 60\% of jack-knife replicates from analysis of $18 \mathrm{~S}$ rRNA matrix (successive weighting of characters, 10 iterations with 100 replicates each, deletion frequency $e^{-1}$ ). Labels indicate jack-knife frequencies. Clades marked with asterisks represent multiple terminals.

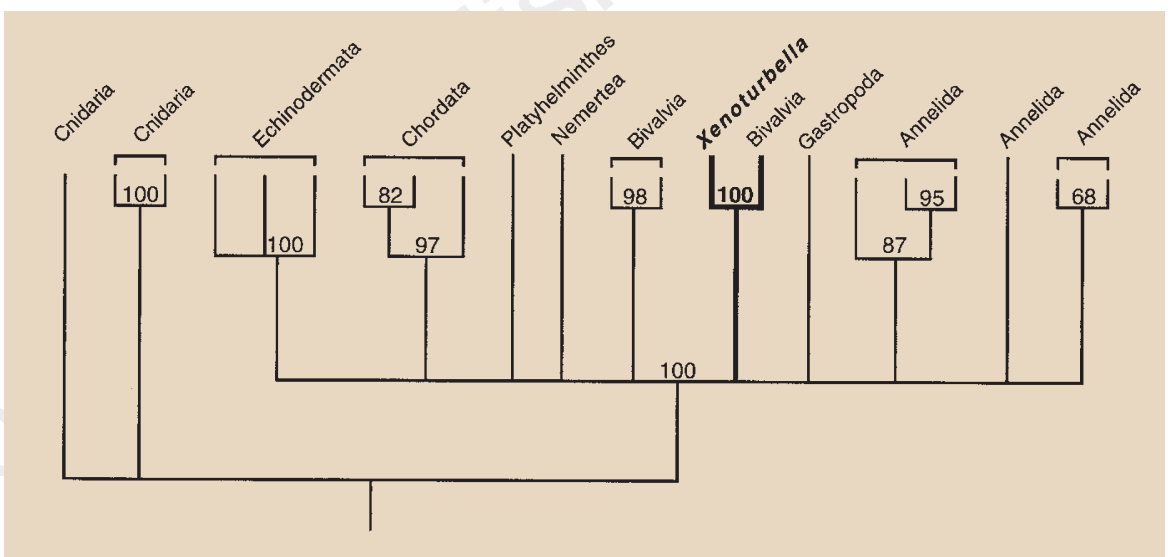

Figure 2 Consensus tree showing groups present in 60\% of jack-knife replicates from analysis of COI matrix $\left(3,000\right.$ replicates, 5 random additions and branch swapping, deletion frequency $\left.e^{-1}\right)$. Labels indicate jackknife frequencies. Full details of tree topology and sequence alignment are available from the authors.

the 18S rRNA sequences, we combined parsimony jack-knifing with successive weighting of characters ${ }^{10}$. We analysed the COI matrix without successive weighting.

The majority-rule consensus tree (Fig. 1) shows groups present in more than $60 \%$ of the jack-knife replicates in the analysis of $18 \mathrm{~S}$ rRNA data. X. bocki is part of a highly supported $(88 \%)$ unresolved clade consisting of representatives of Mollusca, Annelida (including Pogonophora), Echiura, Phoronida, Brachiopoda, Entoprocta, Ectoprocta and Nemertea. The hemichordates, previously suggested as close relations of $X$. bock $i^{1}$ are the sister group of echinoderms (99\%) within a deuterostome clade (63\%). Monophyly of the flatworms, including the acoel Praesagittifera, is supported in all jack-knife replicates, and their sister-group relationship to other bilaterian taxa is also highly supported (94\%). These results are not compatible with hemichordates or platyhelminths as sister groups of $X$. bocki, but suggest that $X$. bocki is a member of the Eutrochozoa (non-moulting protostomes), a clade proposed in previous studies of metazoan evolution on the basis of $18 \mathrm{~S}$ rRNA data ${ }^{11,12}$.
The $60 \%$ majority-rule consensus tree resulting from jack-knife analysis of the COI data (Fig. 2), places X. bocki as part of the Bilateria forming a clade with the protobranch bivalve mollusc Ennucula tenuis in all 3,000 jack-knife replicates. The $X$. bocki COI sequence was translated into amino acids using universal, vertebrate, generalized invertebrate (mollusc), echinoderm, ascidian and flatworm mitochondrial translation codes. However, analysis of the amino-acid sequences did not improve tree resolution, and we found no unambiguous stop codons with any of the codes.

The COI gene appears to evolve too rapidly to be useful in analyses of relationships at phylum or higher levels. Nonetheless, there is a strong indication of a close relationship between $X$. bocki and the bivalve E. tenuis, a clade compatible with the $18 \mathrm{~S}$ rRNA results and supported by similarities in the oogenesis of protobranch bivalves and $X$. bocki described by Israelsson $^{13}$. The same clade (79\%) was present as part of a protostome clade (84\%) excluding flatworms, after analysis without successive weighting of a combined 18S rRNA and 